Check for updates

Cite this: Chem. Sci., 2020, 11, 757

๑ All publication charges for this article have been paid for by the Royal Society of Chemistry

Received 13th November 2019 Accepted 23rd November 2019

DOI: 10.1039/c9sc05741b

rsc.li/chemical-science

\title{
Dandelion flower-like micelles $\uparrow$
}

\author{
Yongchao Yao, (D) $t^{\mathrm{a}}$ Deqiu Xu,,$^{\mathrm{bc}}$ Yuhong Zhu, ${ }^{\mathrm{a}}$ Xin Dai, ${ }^{\mathrm{a}}$ Yunlong $\mathrm{Yu}^{\mathrm{a}}{ }^{a}$ \\ Jianbin Luo (D)*b and Shiyong Zhang (D)*a
}

Dandelion flower-like micelles (DFMs) were prepared by self-assembly of polycaprolactone (PCL) functionalized surface cross-linked micelles (SCMs). Upon reductive stimuli, the SCMs can be released from the DFMs by non-Brownian motion at an average speed of $19.09 \mu \mathrm{m} \mathrm{s}^{-1}$. Similar to the property of dandelion flowers dispersing their seeds over a long distance, the DFMs demonstrated enhanced multicellular tumor spheroid (MTS) penetration, a useful property in the treatment of many diseases including cancer, infection-of-biofilm diseases and ocular problems.

\section{Introduction}

Nature offers inspiration for developing novel functional nanomaterials with desired properties..$^{1-4}$ However, due to the domination of the properties of nanocomposites by their chemical composition and three-dimensional arrangement, the relatively inefficient control of the spatial structure of the nanoscale components usually restrains the optimization of the nano-assembly properties. Biomimetic materials usually only mimic the shape of organisms or only part of their functions. The living organisms capable of structural change in response to the environmental medium are hypnotically fascinating, and more importantly, various special functions are facilitated by these behavioral characteristics. Central to these functions is the unique hierarchical structures of living organisms. ${ }^{3,5}$ For example, dandelion, as a representative flowering plant, has very small plumed seeds assembled together into a larger ball like a flower with spatially well-organized structures. ${ }^{6}$ When they are mature, the plumed seeds easily detach from the flower head, glide by wind power, and disperse over a long distance. This increases the possibility of dispersal of seeds to fertile ground favorable for germination. ${ }^{7}$

Inspired by the unique structure of dandelion flowers and their long-distance dispersal of seeds, we developed dandelion flower-like micelles (DFMs) assembled from polycaprolactone (PCL) functionalized surface cross-linked micelles (SCMs). SCMs are covalently captured small-molecule micelles, which are assembled by amphiphiles with relatively low molecule

${ }^{a}$ National Engineering Research Center for Biomaterials, Sichuan University, 29 Wangjiang Road, Chengdu 610064, China.E-mail: szhang@scu.edu.cn

${ }^{b}$ College of Chemistry and Environmental Protection Engineering, Southwest Minzu University, Chengdu 610041, China. E-mail: luojb1971@163.com

${ }^{c}$ Sichuan Guojian Inspection Co., Ltd., 646000, Luzhou, Sichuan, China

$\dagger$ Electronic supplementary information (ESI) available. See DOI: 10.1039/c9sc05741b

\$ Yongchao Yao and Deqiu Xu contributed equally to this work. weight $(<1000.0 \mathrm{Da}) .^{8-10}$ Featuring facile synthesis, multivalent decoration, high stability or sensitivity to prescribed stimuli, high guest loading capacity etc., SCMs are widely used as platforms for molecular imprinting, ${ }^{11-21}$ drug delivery, ${ }^{9,22}$ fluorescent probe, ${ }^{23}$ light harvesting ${ }^{24,25}$ and catalysis. ${ }^{26-29}$ In this contribution, SCMs were used as the hydrophilic head of amphiphile 1 to build DFMs using a self-assembly process, mimicking the natural structure of dandelion flowers (Scheme 1). After the addition of $20 \mu \mathrm{M}$ of glutathione (GSH), these nanoparticle DFMs can rapidly release SCMs due to electrostatic stress. Furthermore, nanoparticle-tracking analysis (NTA) revealed the occurrence of non-Brownian motion of released SCMs from DFMs with a high directionality of $0.612 \pm 0.129$, which indicates that SCMs can travel over a long distance with an average speed of $19.09 \mu \mathrm{m} \mathrm{s}^{-1}$ (in $2 \mathrm{~min}$ ). Following evaluation in solution, the multicellular tumor spheroid (MTS) model also showed that the DFMs could disperse SCMs over a long distance for deep tumor penetration and effective distribution, which is particularly important for biological applications. As an example of the practical applications, the hydrophobic drug carmofur (HCFU) was incorporated into the

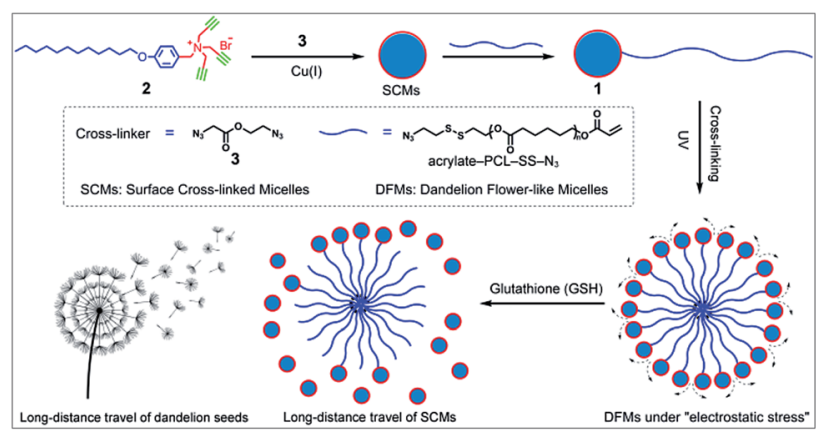

Scheme 1 Synthesis and long-distance dispersal properties of dandelion flower-like micelles (DFMs). The inset shows a sketch of a mature dandelion releasing seeds over a long distance. 
SCM core to create an efficient drug carrier (DFMs@HCFU) for cancer therapy at the MTS level. Benefitting from the longdistance delivery behavior of DFMs, the DFMs@HCFU demonstrated an excellent anti-tumor effect on MTSs. Such a longdistance travel would greatly facilitate tissue penetration of therapeutic reagents, which is highly useful in the treatment of many diseases including cancer, ${ }^{\mathbf{3 0 , 3 1}}$ infection-of-biofilm diseases $^{32,33}$ and ocular problems..$^{34-36}$

\section{Results and discussion}

\section{Synthesis and characterization of the dandelion flower-like micelles (DFMs)}

Previously prepared water-soluble SCMs (see Schemes 1 and S1 and $\mathrm{ESI} \dagger$ for details), with a diameter of $\sim 9 \mathrm{~nm}$, were used as nano-seeds to construct DFMs. ${ }^{8,9}$ As shown in Scheme 1, a hydrophobic biodegradable PCL chain was grafted onto SCMs using click chemistry with a reduction-responsive disulfide spacer. The new surfactant was further assembled into larger DFMs with well-controlled spatial structures. Briefly, we synthesized azide-poly( $\varepsilon$-caprolactone) with a disulfide spacer between PCL and azide (PCL-SS- $\left.\mathrm{N}_{3}\right)$ using 2-[(2-azidoethyl) disulfanyl]ethanol (compound 5; see Scheme S2 in the ESI $\dagger$ ) as an initiator of ring-opening polymerization (ROP). Terminal esterification of PCL-SS- $\mathrm{N}_{3}$ with acryloyl chloride resulted in $\alpha$ azide- $\omega$-acrylate-poly( $\varepsilon$-caprolactone) with a disulfide spacer between PCL and azide (acrylate-PCL-SS- $\mathrm{N}_{3}$; see Scheme S2 in the ESI; Fig. S1, $\left.\dagger M_{\mathrm{n}}=9.3 \mathrm{kDa}, M_{\mathrm{w}} / M_{\mathrm{n}}=1.59\right)$. Acrylate-PCLSS-N $\mathrm{N}_{3}$ was grafted onto the surface of the SCMs by alkyl-azide click chemistry catalyzed by $\mathrm{Cu}(\mathrm{I})$, which resulted in a new amphiphilic conjugate, acrylate-PCL-SS-SCM, that hierarchically assembled into the DFMs with seed micelles (SCMs) located at the surface, similar to dandelion flowers. ${ }^{37-40}$ The number of acrylate-PCL-SS- $\mathrm{N}_{3}$ grafted onto the surface of the SCMs is $\sim 1 / 42$ of that of the surfactant in the SCM, determined by elemental analysis (Table $\mathrm{S} 1 \dagger$ ). Since an SCM contained approximately 40-50 small molecule surfactants according to our previous study, ${ }^{8}$ the SCM on average grafted one acrylatePCL-SS-N $\mathrm{N}_{3}$ group. The core of the DFMs was cross-linked by copolymerization between acrylate, at the end of acrylate-PCLSS- $\mathrm{N}_{3}$, and DVB (divinyl benzene), which was already encapsulated in the core of the DFMs (see the ESI $\dagger$ for details). ${ }^{19}$ Photopolymerization was initiated by the photoinitiator 2,2dimethoxy-2-phenylacetophenone. Following $6 \mathrm{~h}$ of $365 \mathrm{~nm}$ light irradiation, ${ }^{1} \mathrm{H}$ NMR spectroscopy showed the disappearance of most of the protons belonging to alkenyl groups, indicating that copolymerization took place between acrylate groups and DVB (Fig. S2-S3, see the ESI $\dagger$ for the copolymerization mechanism).

The morphology and size of the DFMs were first confirmed by dynamic laser scattering (DLS) and transmission electron microscopy (TEM) analysis. DLS showed a similar size distribution of nanoparticles ( $~ 90 \mathrm{~nm}$ in diameter) before and after UV cross-linking (Fig. 1a). However, the stability of the UV crosslinked DFMs was highly enhanced. After the concentration of DMFs was diluted below the critical micelle concentration (CMC) of 1 in water or DFMs dissolved in tetrahydrofuran (1 mg
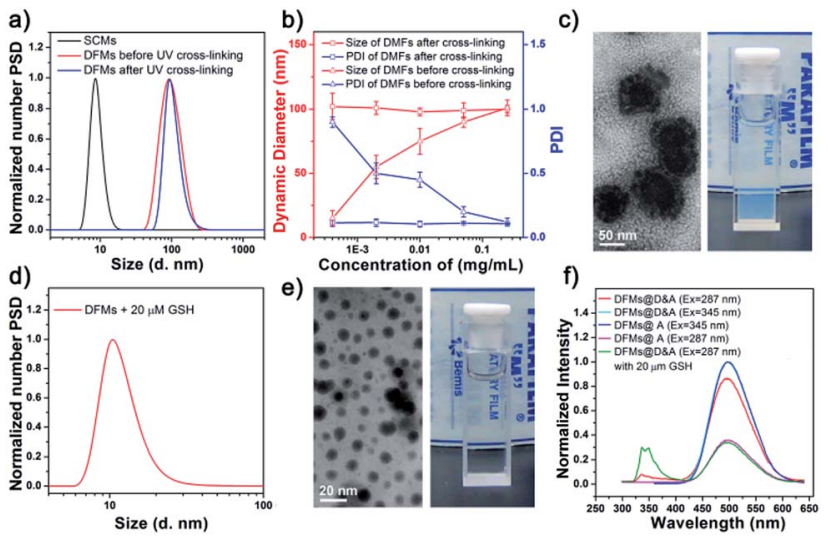

Fig. 1 (a) Distribution of the dynamic diameters of the surface crosslinked micelles (SCMs), and DFMs before and after cross-linking under UV irradiation. (b) Size and particle dispersion index (PDI) of the DFMs after cross-linking or before cross-linking as a function of the concentration of 1 in water. The concentration-independent size indicated the stability of covalently cross-linked DFMs. (c) Transmission electron microscopy (TEM) image (left) and the corresponding photograph (right) of the DFMs in water. (d) Distribution of the hydrodynamic diameters of the DMFs after the addition of $20 \mu \mathrm{M}$ glutathione (GSH) and removal of the precipitate. (e) TEM micrograph (left) and the corresponding photograph (right) of the DFMs after the addition of $20 \mu \mathrm{M}$ GSH and removal of the precipitate. (f) The emission spectra of the naphthalene (D) and dansyl-n-butyl sulfonamide (A) incorporated into the micellar SCMs and the DFM core (DFMs@D\&A) in water, before (red) and after (olive) addition of $20 \mu \mathrm{M} \mathrm{GSH}$.

$\mathrm{mL}^{-1}$ ), the size of the DFMs after cross-linking remained almost unchanged, but almost no reasonable particle size was monitored for the un-cross-linked ones (Fig. 1b, S6 and S7 $\dagger$ ). ${ }^{41-44}$ The TEM images of the DFMs clearly demonstrated the dandelion flower-like structures with seed SCMs located at the shell, acting as dandelion seeds (Fig. 1c). ${ }^{45,46}$

\section{Rapid release of surface cross-linked micelles (SCMs) from DFMs}

To mimic the release of seeds from dandelion flowers by wind, we introduced redox-sensitive disulfides between SCMs and PCL chains, which were cleaved in a reductive environment, releasing the SCMs from the DFMs. ${ }^{4-50}$ To verify this hypothesis, we incubated the DFMs $\left(1 \mathrm{mg} \mathrm{mL}^{-1}\right)$ in the presence of 20 $\mu \mathrm{M}$ GSH to study the structural changes of DFMs and the release of SCMs under the stimuli. As expected, the Tyndall phenomenon rapidly became very weak, accompanied by a small amount of precipitation after the addition of $20 \mu \mathrm{M} \mathrm{GSH}$ (Fig. 1e, the inserted photo; Video S1 $\dagger$ ). DLS and TEM revealed the existence of nanostructures with sizes of $\sim 9 \mathrm{~nm}$, similar to the SCMs (Fig. 1d and e). After dialysis, the nanostructures were subjected to elemental analysis, ${ }^{51,52}$ which showed a similar chemical composition of N, C, and $\mathrm{H}$ as the SCMs (Table S1 $\dagger$ ). Furthermore, ${ }^{1} \mathrm{H}$ NMR spectroscopy of a small amount of the precipitate showed similar peaks to the acrylate-PCL- $\mathrm{N}_{3}$ (Fig. S8†). ${ }^{53}$ Therefore, the release of SCMs from the DFMs upon a specific stimulus can be regarded as the release of seeds from dandelion flowers by wind. 
Further, fluorescence resonance energy transfer (FRET) was used to demonstrate the structural changes of the DFMs and the release of SCMs in real time. Previous reports have established that the distance of the donor-acceptor $(\mathrm{D}-\mathrm{A})$ can be easily distinguished by FRET, in which the transfer efficiency $(E)$ was calculated using the formula $E=R_{0}{ }^{6} /\left(R_{0}{ }^{6}+r^{6}\right)$, where $R_{0}$ is the Förster distance and $r$ is the separation distance between the donor and acceptor. ${ }^{54-56}$ Herein, the naphthalene-dansyl DA pair was chosen in this study. Briefly, naphthalene (D) and dansyl- $n$-butyl sulfonamide (A) were incorporated into the micellar SCMs and the DFM core, respectively (SCMs@D and DFMs@D\&A, see Fig. S9 and S10 and the ESI $\dagger$ for details). FRET was clearly observed in the DFMs@D\&A, with almost no donor emission at $345 \mathrm{~nm}$ and extremely strong acceptor emission at $490 \mathrm{~nm}$ (Fig. 1f, red). Importantly, the FRET phenomenon showed no change over a period of at least five months, suggesting long-term stability of the DFMs; however, FRET almost disappeared after the addition of $20 \mu \mathrm{M}$ GSH, as shown by a decrease in acceptor emission and an increase in donor emission (Fig. 1f, olive). The disappearance in FRET suggests an increase in the D-A distance, consistent with the SCM-releasing behavior of DFMs. ${ }^{57}$ Immediately following the addition of GSH to the mixture, the acceptor emission at $490 \mathrm{~nm}$ dropped quickly. The release rate of the SCMs@D was so fast that the change in acceptor emission was complete (Fig. 1f, olive and magenta). Breaking of disulfide bonds in polymeric micelles often requires a few hours and millimolar concentrations of GSH for completion; ${ }^{\mathbf{5 8 , 5 9}}$ thus, why were the SCMs released from the DFMs so rapidly? Electrostatic repulsion among the SCMs disfavors micellization; the DFM is described as under "electrostatic stress" after assembly (Table $\mathrm{S} 2 \dagger$ ). We have reason to believe that once the hydrophobic force favoring micellization is removed, the nanoparticle DFMs would rapidly release the SCMs due to electrostatic stress, which is reminiscent of that achieved by wind power. According to a previous study, the cleavage of the disulfide bonds in a specially designed micelle takes about 10 minutes. ${ }^{60}$ It is also possible that the electrostatic stress among the SCMs may be the reason for rapid cleavage of disulfide bonds. For a chemical reaction, any stress in the starting reactants, whether conformational, steric, or, in this study, electrostatic, should increase the energy of the ground state, lowering the activation energy of the system. ${ }^{\mathbf{1 0}}$

\section{Long-distance travel of seed SCMs from DFMs}

After the rapid release of SCMs was proven, we demonstrated that the dispersal of the released SCMs from DFMs over a long distance was similar to a dandelion flower dispersing its seeds. NTA with a charge-coupled device camera was used to record in real time the movement of the DFMs (see the ESI $\dagger$ for details). ${ }^{61}$ Indeed, after the addition of $20 \mu \mathrm{M} \mathrm{GSH}$ to $1 \mathrm{~mL}$ of DFMs, the trajectories of the released SCMs from the DFMs showed directional movement and travel for a long distance compared to that of the SCMs that move under Brownian motion in solution in 2 minutes (Fig. 2a, S13 and S14 $\dagger$ ). The directionality of the movement of our DFM system was calculated by comparing Euclidean and accumulated distances. ${ }^{62}$ The larger
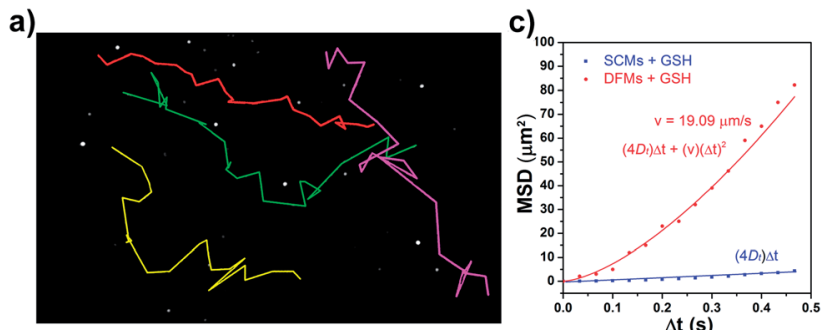

b)
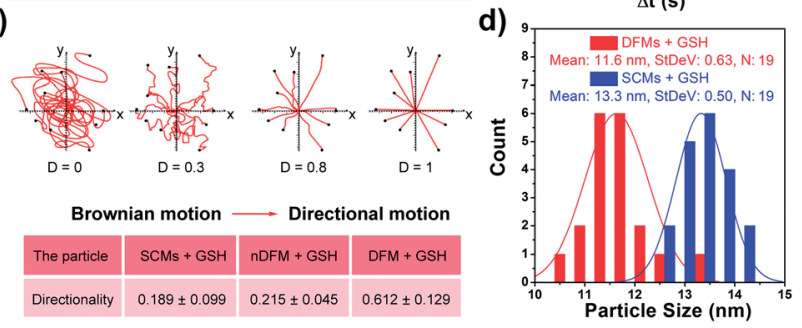

Fig. 2 (a) Nanoparticle-tracking analysis (NTA) trajectories and the video frame of the DFMs after the addition of $20 \mu \mathrm{M} \mathrm{GSH}\left(1 \mathrm{mg} \mathrm{mL}^{-1}\right)$. The colors represent the trajectories of the different SCMs released from the DFMs. (b) Directionality of DFMs and SCMs, and the released SCMs from DFMs. (c) Average mean-square displacements of the released SCMs from the DFMs and the SCMs moving under Brownian motion from the tracking coordinates of 19 particles. (d) Size distribution of the released SCMs from DFMs and SCMs that move under Brownian motion as measured by NTA analysis.

the value of directionality of the particle, the more it tends to move in a straight line. The directionality of the released SCMs from the DFMs was $0.612 \pm 0.129\left(1.0 \mathrm{mg} \mathrm{mL}^{-1}, 20\right.$ particle tracking), which was much higher than that of the SCMs that move under Brownian motion (Fig. 2b). Although the directionality is not a direct parameter for judging chemotaxis, it can be used to characterize the straightness of movement. The mean-square displacement (MSD) versus time was also plotted to give more insight into the movement mechanism of the DFMs (Fig. 2c). The average MSD of the 20 nanoparticles indicated directional motion of the SCMs released from the DFMs (Fig. 2c, S15 and S16 ${ }^{\dagger}$ ). The average speed ( $v$ ) of the SCMs released from the DFMs was $19.09 \mu \mathrm{m} \mathrm{s}^{-1}$ according to the equation $r^{2}=4 D_{\mathrm{t}} \Delta t+(v \Delta t)^{2}\left(D_{\mathrm{t}}\right.$ is the particle diffusion coefficient, Fig. S17†). ${ }^{63}$ Moreover, the 'apparent' size of the SCMs released from the DFMs was smaller than that of the same structures moving under Brownian motion (Fig. 2d). The 'apparent' size and diffusion of the nanoparticle are inversely proportional according to the Stokes-Einstein equation $\left(D_{\mathrm{t}}=\right.$ $T K_{\mathrm{B}} / 3 \pi \eta d$, where $K_{\mathrm{B}}$ is the Boltzmann constant, $T$ is the temperature, $\eta$ is the viscosity and $d$ is the hydrodynamic diameter). Thus, this suggested that the movement speed of the SCMs released from the DFMs is considerably fast. Such highspeed directional movement indicates the occurrence of nonBrownian motion, which demonstrates that the released SCMs from the DFMs can travel over a long distance similar to a dandelion flower dispersing its seeds. It is reasonable to expect that once the hydrophobic force constraint is removed under a given stimulus, the satellite micelle SCMs would be launched due to electrostatic repulsion and get a great initial speed for a long travel distance. This design is much different 
from the traditional assemblies, where the relatively inefficient control of the spatial structure of the nanoscale components restricts the optimization of the nano-assembly properties.

Following evaluation in solution, MTSs derived from human lung carcinoma (A549) cells were employed as a 3D tumor model to evaluate the long-distance travel ability of the released SCMs from the DFMs ${ }^{64-66}$ Briefly, the hydrophobic dye Nile Red (NR) was incorporated into the SCM core (SCMs@NR) of the DFMs, enabling easy monitoring of the distribution of SCMs in real-time (DFMs@NR, see the ESI $\dagger$ for details). As a control, dandelion flower-like micelles lacking the stimulus-response activity (nDFMs) were prepared by a similar synthesis procedure using acrylate-PCL- $\mathrm{N}_{3}$ (see Scheme S3 and Fig. S1 in the ESI; $\dagger$ $\left.M_{\mathrm{n}}=8.9 \mathrm{kDa}, M_{\mathrm{w}} / M_{\mathrm{n}}=1.73\right)$ as the hydrophobic segment instead of acrylate-PCL-SS-N $\mathrm{N}_{3}$. As shown in Fig. $\mathrm{S} 5$ and $\mathrm{S} 6, \uparrow$ the size distribution, morphology, and critical micelle concentration (CMC) of the nDFMs were essentially the same as those of the DFMs. Subsequently, nDFM labeled NR (nDFMs@NR)was prepared using a similar synthesis procedure to that of the DFMs@NR (Table S2, see the ESI $\dagger$ for details). Then the MTSs were incubated with DFMs@NR, nDFMs@NR, or SCMs@NR $\left(20 \mu \mathrm{g} \mathrm{mL} \mathrm{m}^{-1}, \mathrm{NR}\right)$ for $3 \mathrm{~h}$, followed by incubation in the presence of $20 \mu \mathrm{M} \mathrm{GSH}$ for $24 \mathrm{~h}$ (see the ESI $\dagger$ for details), ${ }^{22,43}$ after which they were washed in phosphate buffer saline buffer and detected by light sheet fluorescence microscopy (LSFM). ${ }^{67}$ As compared in Fig. 3, with the nDFMs@NR, each equatorial section of A549 MTS showed low fluorescence signals, revealing limited penetration. In contrast, high fluorescence signals were detected in the MTS equatorial sections following incubation with DFMs@NR, indicating enhanced tumor penetration. Interestingly, the penetration capability of DFMs@NR at $20 \mu \mathrm{M}$ GSH was stronger than that of SCMs@NR, presumably as a result of the rapid release and long travel distance of the released SCMs induced by electrostatic stress. ${ }^{5}$ Moreover, the differences in the permeability of different nanoparticles are clearly shown by the traces showing the fluorescence intensity recorded along the regions of interest (ROIs) in the randomly traced diameter of each section (Fig. 3). Furthermore, quantitative cellular uptake analysis indicated robust penetration of the DFMs@NR at $20 \mu \mathrm{M}$ GSH, verifying that almost all tumor cells (94.3\%) in the MTSs took in NR, which was 2.8- and 1.2fold higher than that with the nDFMs@NR (33.8\%) and SCMs@NR (81.9\%), respectively (Fig. 4).

\section{Application of DFMs as platforms for cancer therapy}

Such a long-distance travel behavior is particularly important for biological applications. As an example of the practical applications, the DFMs were further employed as platforms for cancer therapy at the MTS level. ${ }^{68}$ Instead of NR, the HCFU was incorporated into the SCM core of the DFMs and nDFMs, respectively (DFMs@HCFU; nDFMs@HCFU, see Fig. S19-S21†). As shown in Fig. 5a, only a few ethidium homodimer-positive cells were observed in the external region of the MTSs in the nDFMs@HCFU-treated group (11.36 $\mu \mathrm{g} \mathrm{mL}^{-1}$ HCFU) following a $48 \mathrm{~h}$ incubation, which was due to the poor penetration of the nDFMs. Nevertheless, cell death was high following treatment

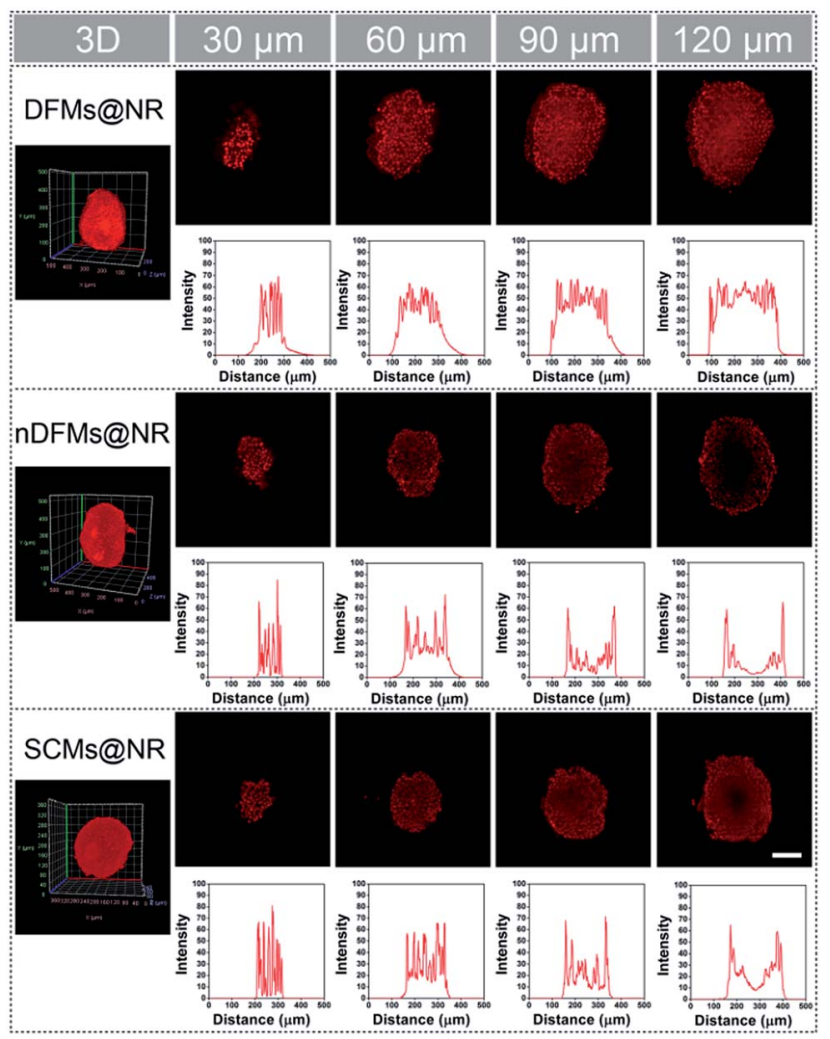

Fig. 3 Penetration of DFMs@NR, nDFMs@NR, and SCMs@NR into A549 multicellular tumor spheroids (MTSs) after $24 \mathrm{~h}$ of incubation determined by light sheet fluorescence microscopy (LSFM). For each sample, images from the $30 \mu \mathrm{m}$ to $120 \mu \mathrm{m}$ section of the MTSs show a red channel $\left(\lambda_{\mathrm{ex}}=540 \mathrm{~nm}\right.$ ) and the corresponding intensity profile of regions of interest (ROIs) in the randomly traced diameter of each section. Scale bar: $100 \mu \mathrm{m}$.

with the DFMs@HCFU, indicating enhancement of the penetration of DFMs@HCFU. Subsequently, the cell viability of the spheroids was assessed using the MTS assay. ${ }^{69}$ As shown in Fig. 5b, cell viability in MTSs co-cultured with DFMs@HCFU for $48 \mathrm{~h}$ was very low, while the nDFMs@HCFU was less cytotoxic for MTSs under the same conditions. The MTS growth profiles for 4 days are summarized in Fig. $5 \mathrm{c}$ and d. The nDFMs@HCFU
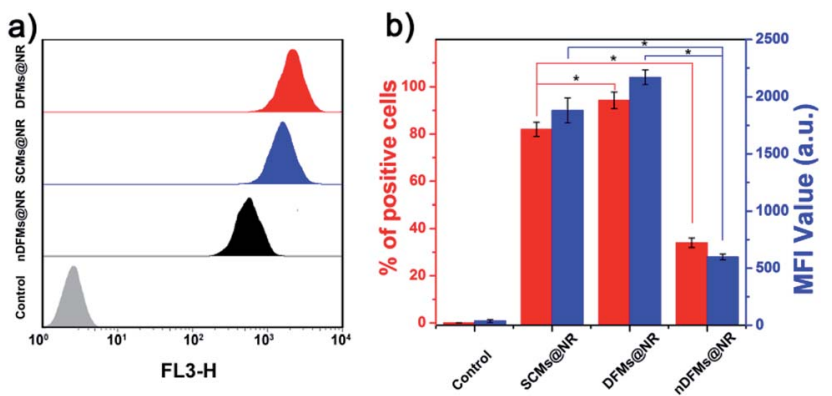

Fig. 4 (a) The flow cytometric profiles and (b) Nile Red (NR) mean fluorescence intensity (MFI) and percentage of NR-positive cells in A549 MTSs as quantitated by flow cytometry (mean \pm SD, $n=3, * p<$ 0.05). 

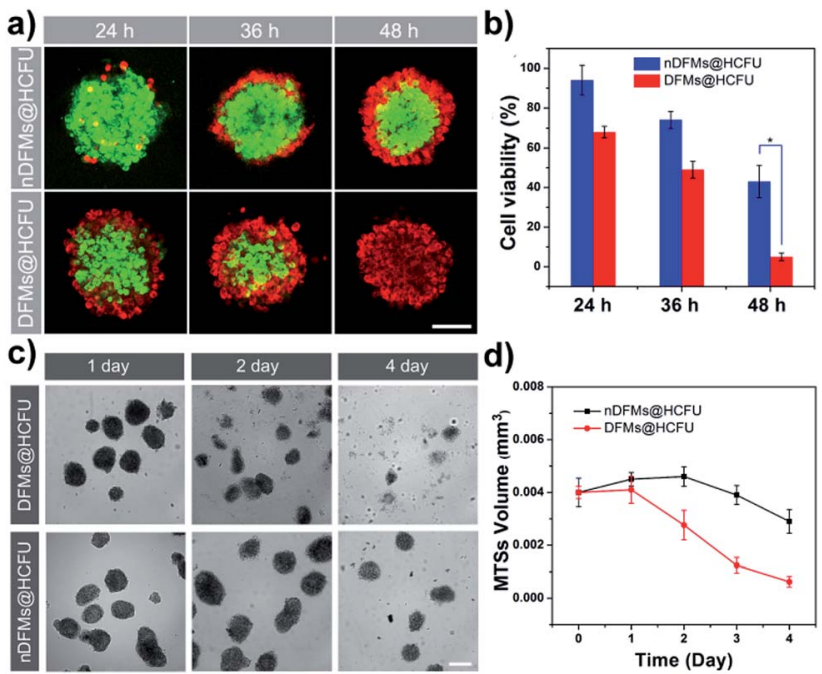

Fig. 5 (a) Images of the spheroids stained using the LIVE/DEAD $®$ kit from confocal laser scanning microscopy (CLSM), (scale bar: $75 \mu \mathrm{m}$ ). (b) Viability of MTSs incubated for $24 \mathrm{~h}, 36 \mathrm{~h}$ and $48 \mathrm{~h}$ with $11.36 \mu \mathrm{g}$ $\mathrm{mL}^{-1}$ hydrophobic drug carmofur (HCFU) delivered by different formulations (mean $\pm \mathrm{SD}, n=5, * p<0.05$ ). (c) Photographs of A549 MTS treatment with DFMs@HCFU (up) and nDFMs@HCFU (down) on the 1st, 2nd and 4th days (scale bar: $200 \mu \mathrm{m}$ ). (d) A549 MTS growth profiles ( 4 day-time course) following a single administration of the drug nanocarrier (mean $\pm \mathrm{SD}, n=15$ ).

slightly inhibited MTS growth, and the MTSs showed dense structures and were spherical in shape after 4 days of treatment. Nevertheless, MTSs incubated with DFMs@HCFU emerged with a lot of detached cells and a seriously damaged morphology. It should be noted that the DFMs and nDFMs showed negligible cytotoxicity against A549 cells even at a concentration of $0.96 \mathrm{mg}$ $\mathrm{mL}^{-1}$ (Fig. S22 $\dagger$ ), suggesting that the cytotoxicity of DFMs@HCFU and nDFMs@HCFU was caused by the enclosed HCFU rather than the DFMs and nDFMs themselves. ${ }^{70}$ It should also be emphasized that the above results not only verified that the DFMs have promising applications, but they also revealed the structure and function of the DFMs from another point of view.

\section{Conclusions}

In conclusion, DFMs with spatially well-organized structures were developed. Upon reductive stimuli, the SCMs achieved a speedy release and long-distance travel due to the electrostatic repulsion (electrostatic stress) among the satellite SCMs, just like the behavior of dandelion seeds powered by wind. By taking advantage of the long-distance delivery of the DFMs, DFMs@HCFU demonstrated an excellent anti-tumor effect on MTSs. Compared to the saline-treated group, the tumor inhibitory rate of DFMs@HCFU after 4 days of treatment was calculated to be up to $84.9 \pm 1.6 \%$, which is much more efficient than that of nDFMs@HCFU (41.4 $\pm 4.6 \%)$. With their well-organized spatial structure and long-distance delivery capacity, DFMs are expected to have important applications in the treatment of many diseases including cancer, infection-of-biofilm diseases and ocular problems.

\section{Conflicts of interest}

There are no conflicts to declare.

\section{Acknowledgements}

The authors are grateful for financial support from the National Natural Science Foundation of China (No. 21975165 and 51673130). The authors also thank the Center of Testing and Analysis, Sichuan University, for TEM and NMR measurements.

\section{Notes and references}

1 V. P. Terrier, H. Adihou, M. Arnould, A. F. Delmas and V. Aucagne, Chem. Sci., 2016, 7, 339-345.

2 B. Su, Y. Tian and L. Jiang, J. Am. Chem. Soc., 2016, 138, 17271748.

3 Q. a. Meng, Q. Wang, K. Zhao, P. Wang, P. Liu, H. Liu and L. Jiang, Adv. Funct. Mater., 2016, 26, 7378-7385.

4 P. Tseng, B. Napier, S. Zhao, A. N. Mitropoulos, M. B. Applegate, B. Marelli, D. L. Kaplan and F. G. Omenetto, Nat. Nanotechnol., 2017, 12, 474.

5 H. Sun, Q. Luo, C. Hou and J. Liu, Nano Today, 2017, 14, 1641.

6 Q. Meng, Q. Wang, H. Liu and L. Jiang, NPG Asia Mater., 2014, 6, e125.

7 C. Cummins, M. Seale, A. Macente, D. Certini, E. Mastropaolo, I. M. Viola and N. Nakayama, Nature, 2018, 562, 414-418.

8 S. Zhang and Y. Zhao, Macromolecules, 2010, 43, 4020-4022.

9 S. Zhang and Y. Zhao, J. Am. Chem. Soc., 2010, 132, 1064210644.

10 Y. Zhao, Langmuir, 2016, 32, 5703-5713.

11 J. K. Awino, R. W. Gunasekara and Y. Zhao, J. Am. Chem. Soc., 2016, 138, 9759-9762.

12 J. K. Awino, R. W. Gunasekara and Y. Zhao, J. Am. Chem. Soc., 2017, 139, 2188-2191.

13 J. K. Awino, L. Hu and Y. Zhao, Org. Lett., 2016, 18, 16501653.

14 J. K. Awino and Y. Zhao, J. Am. Chem. Soc., 2013, 135, 1255212555.

15 J. K. Awino and Y. Zhao, Chem. Commun., 2014, 50, 57525755.

16 J. K. Awino and Y. Zhao, Chem.-Eur. J, 2015, 21, 655-661.

17 S. Fa and Y. Zhao, Chem. Mater., 2017, 29, 9284-9291.

18 S. Fa and Y. Zhao, Chem.-Eur. J, 2018, 24, 150-158.

19 R. W. Gunasekara and Y. Zhao, J. Am. Chem. Soc., 2017, 139, 829-835.

20 R. W. Gunasekara and Y. Zhao, Org. Lett., 2017, 19, 41594162.

21 Y. Zhao, Chem.-Eur. J, 2018, 24, 14001-14009.

22 Y. Chen, J. Huang, S. Zhang and Z. Gu, Chem. Mater., 2017, 29, 3083-3091.

23 Y.-Z. Chen, P.-Z. Chen, H.-Q. Peng, Y. Zhao, H.-Y. Ding, L.-Z. Wu, C.-H. Tung and Q.-Z. Yang, Chem. Commun., 2013, 49, 5877-5879. 
24 H.-Q. Peng, Y.-Z. Chen, Y. Zhao, Q.-Z. Yang, L.-Z. Wu, C.-H. Tung, L.-P. Zhang and Q.-X. Tong, Angew. Chem., Int. Ed., 2012, 51, 2088-2092.

25 G. Chadha, Q.-Z. Yang and Y. Zhao, Chem. Commun., 2015, 51, 12939-12942.

26 M. D. Arifuzzaman and Y. Zhao, ACS Catal., 2018, 8, 81548161.

27 G. Chadha and Y. Zhao, Chem. Commun., 2014, 50, 27182720.

28 S. Zhang and Y. Zhao, Chem. Commun., 2012, 48, 999810000.

29 G. Chadha and Y. Zhao, J. Colloid Interface Sci., 2013, 390, 151-157.

30 Q. Sun, X. Sun, X. Ma, Z. Zhou, E. Jin, B. Zhang, Y. Shen, E. A. Van Kirk, W. J. Murdoch, J. R. Lott, T. P. Lodge, M. Radosz and Y. Zhao, Adv. Mater., 2014, 26, 7615-7621.

31 H.-J. Li, J.-Z. Du, J. Liu, X.-J. Du, S. Shen, Y.-H. Zhu, X. Wang, X. Ye, S. Nie and J. Wang, ACS Nano, 2016, 10, 6753-6761.

32 T.-O. Peulen and K. J. Wilkinson, Environ. Sci. Technol., 2011, 45, 3367-3373.

33 F. Liu, D. He, Y. Yu, L. Cheng and S. Zhang, Bioconjugate Chem., 2019, 30, 541-546.

34 R. Tong, H. D. Hemmati, R. Langer and D. S. Kohane, J. Am. Chem. Soc., 2012, 134, 8848-8855.

35 D. A. Srinivasarao, G. Lohiya and D. S. Katti, WIREs: Nanomed. Nanobiotechnol., 2018, e1548.

36 A. Than, C. Liu, H. Chang, P. K. Duong, C. M. G. Cheung, C. Xu, X. Wang and P. Chen, Nat. Commun., 2018, 9, 4433.

37 L.-J. Chen and H.-B. Yang, Acc. Chem. Res., 2018, 51, 26992710.

38 L.-J. Chen, Y.-Y. Ren, N.-W. Wu, B. Sun, J.-Q. Ma, L. Zhang, H. Tan, M. Liu, X. Li and H.-B. Yang, J. Am. Chem. Soc., 2015, 137, 11725-11735.

39 B. Sun, M. Wang, Z. Lou, M. Huang, C. Xu, X. Li, L.-J. Chen, Y. Yu, G. L. Davis, B. Xu, H.-B. Yang and X. Li, J. Am. Chem. Soc., 2015, 137, 1556-1564.

40 C. Wu, L. Lei, X. Zhu, J. Yang and Y. Xie, Small, 2007, 3, 15181522.

41 C. Liao, X. Dai, Y. Chen, J. Liu, Y. Yao and S. Zhang, Adv. Funct. Mater., 2019, 29, 1806567.

42 C. Li, J. Zhang, S. Zhang and Y. Zhao, Angew. Chem., Int. Ed., 2019, 58, 1643-1647.

43 J. Huang, Y. Yu, L. Wang, X. Wang, Z. Gu and S. Zhang, ACS Appl. Mater. Interfaces, 2017, 9, 29030-29037.

44 X. Ma, J. Liu, L. Lei, H. Yang and Z. Lei, J. Appl. Polym. Sci., 2019, 136, 47946.

45 X. Miao, H. Liao, Z. Deng, C. Li, T. Wu, H. Zhang, M. Liu, X. Cheng and X. Wang, ACS Biomater. Sci. Eng., 2017, 3, 2259-2266.

46 L. Y. T. Chou, F. Song and W. C. W. Chan, J. Am. Chem. Soc., 2016, 138, 4565-4572.

47 X. Liu, W. Lin, D. Astruc and H. Gu, Prog. Polym. Sci., 2019, 96, 43-105.
48 N. Drude, O. H. Winz, F. M. Mottaghy, M. Roller, H. Königs, M. Möller, S. Singh and A. Morgenroth, Small, 2018, 14, 1704093.

49 C. Luo, J. Sun, B. Sun, D. Liu, L. Miao, T. J. Goodwin, L. Huang and Z. He, Small, 2016, 12, 6353-6362.

50 C. H. Sohn, J. Gao, D. A. Thomas, T.-Y. Kim, W. A. Goddard III and J. L. Beauchamp, Chem. Sci., 2015, 6, 4550-4560.

51 P. A. Zhizhko, A. V. Pichugov, N. S. Bushkov, F. Allouche, A. A. Zhizhin, D. N. Zarubin and N. A. Ustynyuk, Angew. Chem., Int. Ed., 2018, 57, 10879-10882.

52 M. Humood, Y. Shi, M. Han, J. Lefebvre, Z. Yan, M. Pharr, Y. Zhang, Y. Huang, J. A. Rogers and A. A. Polycarpou, Small, 2018, 14, 1703852.

53 M. Ding, J. Li, X. He, N. Song, H. Tan, Y. Zhang, L. Zhou, Q. Gu, H. Deng and Q. Fu, Adv. Mater., 2012, 24, 3639-3645. 54 C. Ma, F. Zeng, L. Huang and S. Wu, J. Phys. Chem. B, 2011, 115, 874-882.

55 J. Hu, L. Dai and S. Liu, Macromolecules, 2011, 44, 4699-4710. 56 L. Wang, R. Yan, Z. Huo, L. Wang, J. Zeng, J. Bao, X. Wang, Q. Peng and Y. Li, Angew. Chem., Int. Ed., 2005, 44, 60546057.

57 Y. Zhao and Z. Zhong, J. Am. Chem. Soc., 2005, 127, 1789417901.

58 A. N. Koo, H. J. Lee, S. E. Kim, J. H. Chang, C. Park, C. Kim, J. H. Park and S. C. Lee, Chem. Commun., 2008, 6570-6572.

59 L. Zhang, W. Liu, L. Lin, D. Chen and M. H. Stenzel, Biomacromolecules, 2008, 9, 3321-3331.

60 Y. Yao, H. Xu, C. Liu, Y. Guan, D. Xu, J. Zhang, Y. Su, L. Zhao and J. Luo, RSC Adv., 2016, 6, 9082-9089.

61 L. K. E. A. Abdelmohsen, M. Nijemeisland, G. M. Pawar, G.-J. A. Janssen, R. J. M. Nolte, J. C. M. van Hest and D. A. Wilson, ACS Nano, 2016, 10, 2652-2660.

62 P. Morin, G. Wickman, J. Munro, G. J. Inman and M. F. Olson, Eur. J. Cell Biol., 2011, 90, 13-25.

63 S. J. Ebbens and J. R. Howse, Langmuir, 2011, 27, 1229312296.

64 Y. Li, X. Xu, X. Zhang, Y. Li, Z. Zhang and Z. Gu, ACS Nano, 2017, 11, 416-429.

65 W. Dai, H. Dong, K. Guo and X. Zhang, Chem. Sci., 2018, 9, 1753-1759.

66 G. Lu, C. Lv, W. Bao, F. Li, F. Zhang, L. Zhang, S. Wang, X. Gao, D. Zhao, W. Wei and H.-y. Xie, Chem. Sci., 2019, 10, 4847-4853.

67 G. Lazzari, D. Vinciguerra, A. Balasso, V. Nicolas, N. Goudin, M. Garfa-Traore, A. Fehér, A. Dinnyés, J. Nicolas, P. Couvreur and S. Mura, Eur. J. Pharm. Biopharm., 2019, 142, 195-203.

68 Y. Li, X. Zhang, Z. Zhang, H. Wu, X. Xu and Z. Gu, Mater. Horizons, 2018, 5, 1047-1057.

69 E. Gaio, D. Scheglmann, E. Reddi and F. Moret, J. Photochem. Photobiol., 2016, 161, 244-252.

70 W. Wu, S. Li, Z. Lin and J. Li, J. Control. Release, 2015, 213, e111-e112. 